\title{
Kunststoffgleitpaarungen in der Fördertechnik - Methoden zur Messung von Reibungswerten
}

\author{
PROF. DR.-ING. KLAU NENDEL, \\ DIPL.-ING. FRANK MITZSCHKE \\ TECHNISCHE UNIVERSITÄT CHEMNITZ, PROFESSUR FÖRDERTECHNIK
}

\section{Zusammenfassung}

Stückgutförderer mit umlaufenden Zugmitteln lassen sich auf ein einheitliches Modell reduzieren. In den aus diesem Einheitsmodell abgeleiteten Kontaktpaarungen treten zwischen den einzelnen Elementen des Fördersystems Reibung und somit Reibungskräfte auf. Für die Konstruktion und den wirtschaftlichen Betrieb der Fördersysteme ist die Höhe der Reibungswerte entscheidend. Diese sind keine Materialkennwerte, sie können nur experimentell bestimmt werden. Die existierenden Messeinrichtungen sind dafür jedoch ungeeignet. Aus diesem Grunde wurde ein neuer Versuchsstand entwickelt, auf dem Probekörper und Bauteile mit variablen Formen und Abmessungen getestet werden können. Erste Messungen mit dem neuen Versuchsstand haben neben den Abhängigkeiten des Reibungswertes von der Geschwindigkeit und der Normalkraft auch aufgezeigt, dass praxisrelevante Reibungswertmessungen als Dauerversuche durchgeführt werden müssen.

\begin{abstract}
All conveying systems for unit loads with tractions means are based on a uniform model. The components of this uniform conveying system are contacting each other. Those contacts produce friction and friction force. The extent of the coefficient of friction is important for the design and the economic efficiency of the conveying system. The coefficient of friction is not material characteristic and therefore, needs to be qualified by an experiment. Already existing experimental rigs are inapplicable. On this account it was necessary to develop a new experimental rig to test samples and components of variable forms and dimensions. First measurements show dependency between the coefficient of friction and the velocity, and the normal force, respectively. The tests also point out that friction tests need to be carried out by long term tests.
\end{abstract}

\section{Einleitung}

Bei der Entwicklung von Elementen für Stückgutförderer spielen Reibungswerte eine große Rolle. Sie bestimmen nicht nur die notwendige Antriebsleistung, sondern begrenzen die Leistungsfähigkeit der Fördersysteme im Allgemeinen. Konstruktiv wird deshalb versucht, die Reibungswerte durch Modifikation der am Kontakt beteiligten Kunststoffbauteile, d.h. durch den Einsatz neuer Materialien, zu beeinflussen. Der Erfolg dieser Materialmodifikationen muss anschließend experimentell bestätigt werden. Dafür werden einfache und schnelle Messmethoden benötigt, die sowohl komplette Bauteile als auch Probekörper mittlerer Abmessungen unter praxisrelevanten Bedingungen testen können. Mit den gegenwärtigen Versuchsständen ist dies jedoch nicht möglich.

\section{2. $\quad$ Einheitsmodell eines Stückgutförderers mit Zugmittel}

Aus der in Abbildung 1 dargestellten Systematik der Stetigförderer für Stückgüter sind fünf Hauptgruppen zu erkennen, wobei den Stetigförderern mit umlaufenden Zugmitteln die größte Bedeutung zukommt. 


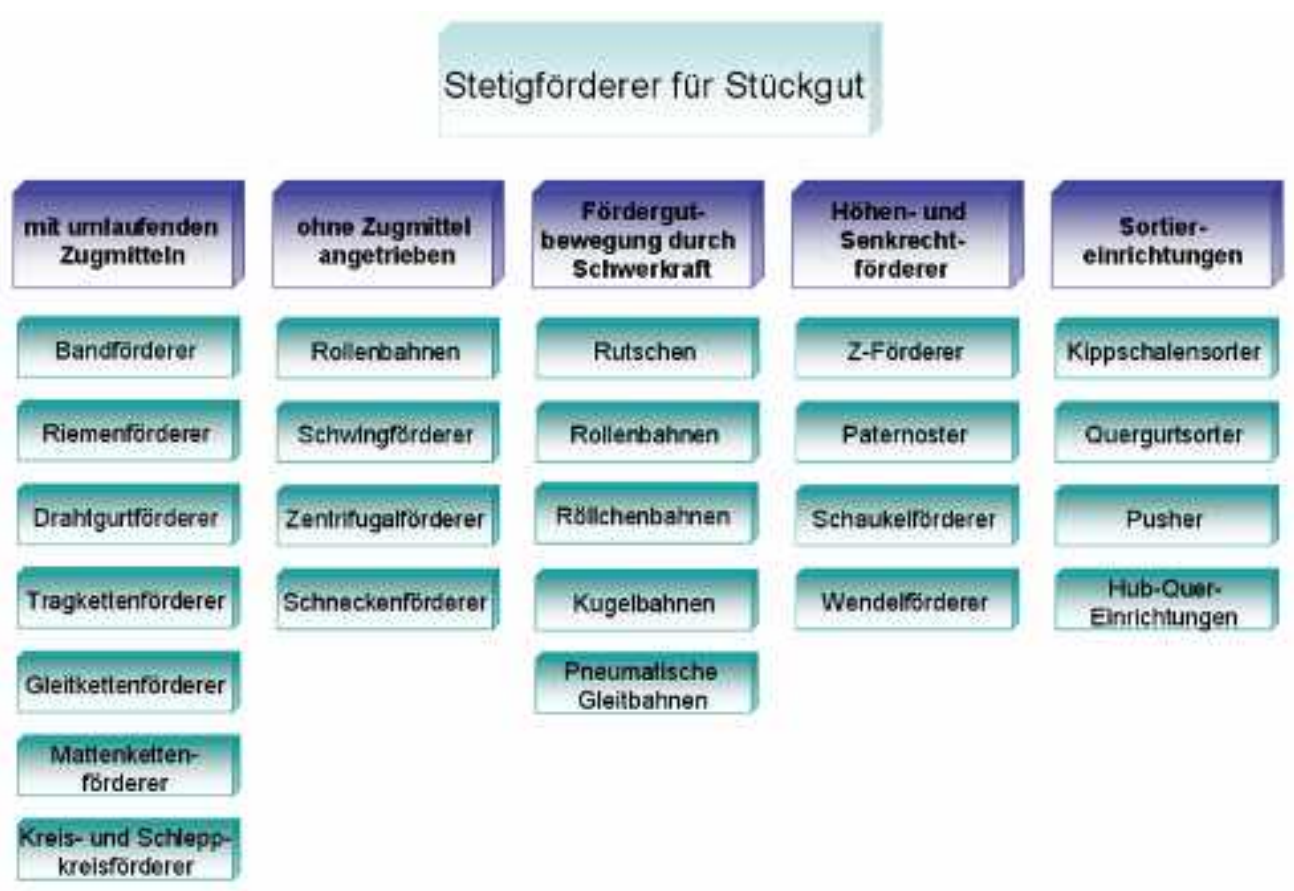

Abbildung 1: Systematik der Stetigförderer für Stückgüter

Das besondere Merkmal dieser Gruppe ist, dass die zu transportierenden Stückgüter direkt auf dem Zugmittel aufliegen und dass das Zugmittel selbst die Transportstrecke darstellt. Trotz der Mannigfaltigkeit lassen sich alle zugmittelgebundene Stetigförderer zu einem in Abbildung 2 dargestellten einheitlichen Modell vereinfachen.

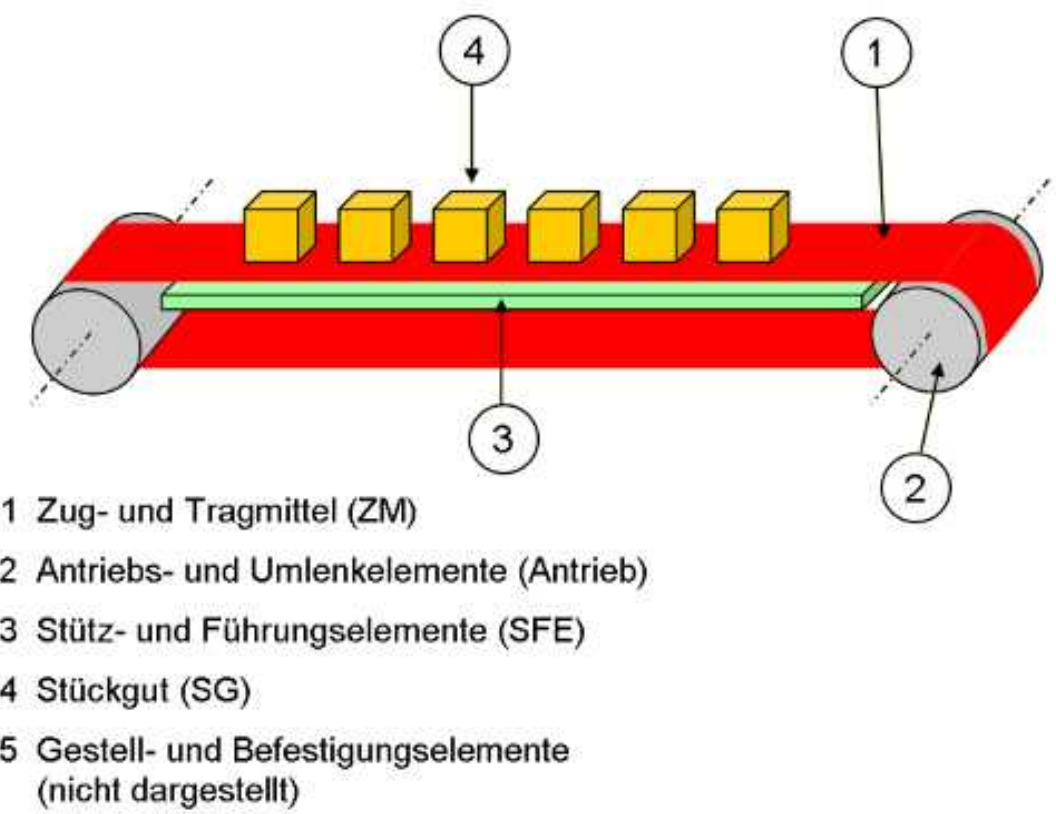

Abbildung 2: Modell zugmittelgebundener Stetigförderer

Zentrales Element dieses Einheitsmodells ist das endlose, umlaufende Zugmittel, welches die Aufgaben hat, das zu transportierende Stückgut zu tragen und von der Aufnahme- zur Abgabestelle zu ziehen. Damit das Zugmittel geführt wird und es die Masse des Stückgutes aufnehmen kann, gleitet dieses auf Stütz- und Führungselementen. Sie führen das Zugmittel und definieren somit die Geometrie der Förderstrecke. Die Aufgabe der Antriebs- und Umlenkelemente besteht darin, das Zugmittel anzutreiben bzw. je nach Streckenverlauf horizontal oder vertikal umzulenken. Die Lage des Antriebes muss sich nicht zwangsläufig an der Stirnseite des Fördersystems befinden, Mittenantriebe sind ebenfalls denkbar. Da das Fördersystem nicht frei im Raum schweben kann, ist es auf Gestell- und Befestigungselementen aufgeständert. 
Ausgehend von diesem Einheitsmodell lassen sich für zugmittelgebundene Stückgutförderer folgende Kontaktstellen zwischen den einzelnen Elementen des Einheitsmodells ableiten:

- Zugmittel - Stütz- und Führungselemente

- Zugmittel - Stückgut

- $\quad$ Zugmittel - Antriebs- und Umlenkelemente

\section{Bedeutung der Reibung}

An den Kontaktstellen berühren sich die jeweiligen Funktionselemente des Fördersystems, wobei es zu einer Relativbewegung zwischen diesen kommt. Dabei entstehen Reibungskräfte, die den Bewegungen und somit den Bewegungsrichtungen entgegen wirken. Diese Reibungskräfte haben große Auswirkungen auf die Funktion und Leistungsfähigkeit der Fördersysteme und dürfen nicht vernachlässigt werden. Die folgenden Beispiele sollen dies verdeutlichen.

\section{1. $\quad$ Reibung zwischen Zugmittel und den Stütz- und Führungselementen}

Zur Dimensionierung des Antriebes eines Fördersystems wird eine Antriebsleistung $\mathrm{P}_{\mathrm{A}}$ benötigt. Diese berechnet sich z.B. bei Gleitkettenfördersystemen (vgl. Abb. 3) aus der anliegenden Zugkraft $\mathrm{F}_{Z}$ im Zugmittel und der Fördergeschwindigkeit v :

$$
\mathrm{P}_{\mathrm{A}}=\mathrm{F}_{\mathrm{Z}} \cdot \mathrm{V}
$$

Die Zugkraft im Zugmittel entsteht durch die Reibung zwischen Zugmittel und den Stütz- und Führungselementen. An der Umlenkung ist die Zugkraft nahezu Null, steigt aber zum Antrieb hin kontinuierlich an. Diverse Streckenelemente wie Kurvenräder oder Gleitbögen verstärken diesen Anstieg. (vgl. [Auerbach06])

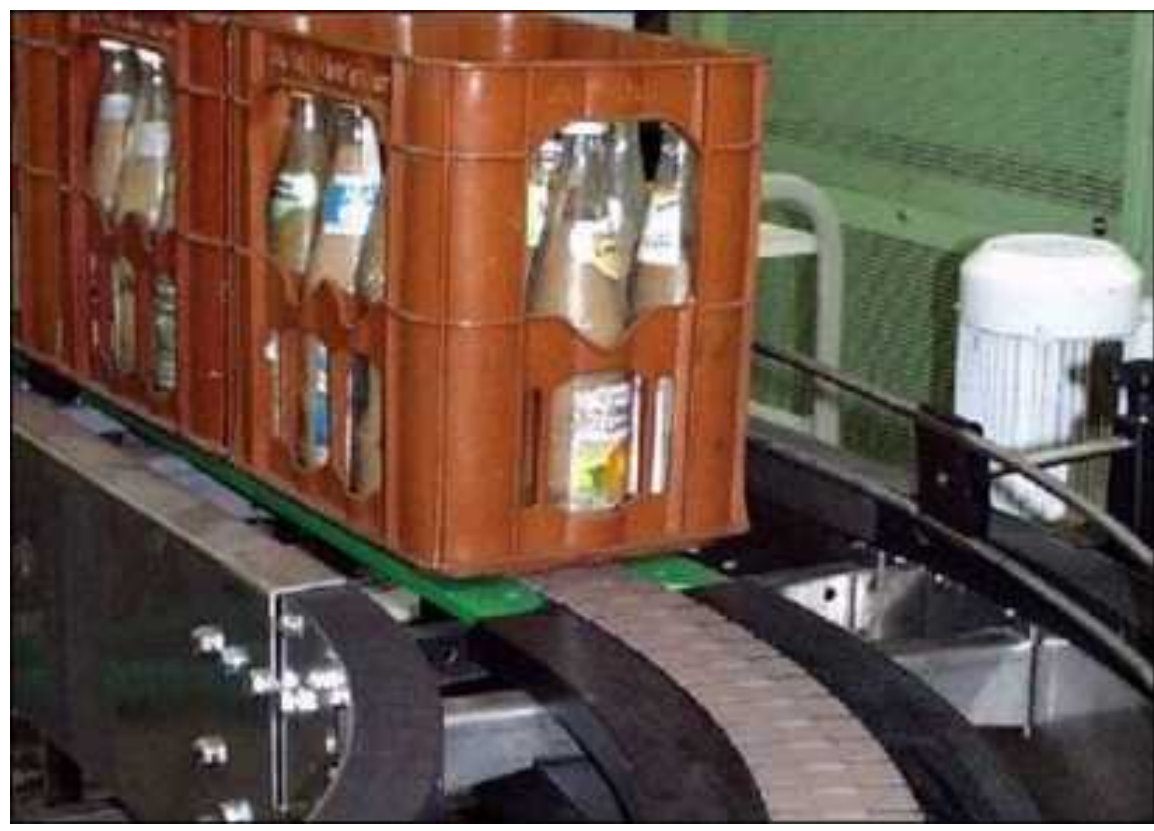

Abbildung 3: Gleitkettenfördersystem

Für gerade Steckenabschnitte berechnet sich die Zugkraft aus:

$$
\mathrm{F}_{\mathrm{Z}}=\mathrm{L}\left(\mathrm{q}_{\mathrm{SG}}+\mathrm{q}_{\mathrm{ZM}}\right) \cdot \mathrm{g} \cdot \mu_{\mathrm{ZM}-\mathrm{SFE}}
$$

L ist die Länge des betrachteten Förderstreckenabschnittes, q die spezifische Masse des Stückgutes bzw. des Zugmittels in kg/m und $\mu_{\mathrm{ZM}-\mathrm{SFE}}$ der Reibwert zwischen dem Zugmittel und den Stütz- und Führungselementen. 
Aus wirtschaftlichen Gründen wird für Fördersysteme eine geringe Antriebsleistung oder eine besonders lange Förderstrecke pro Antrieb gefordert. Dies kann nur durch den Einsatz von Materialkombinationen mit geringem Reibungswert realisieren werden.

\section{2. $\quad$ Reibung zwischen Zugmittel und Stückgut}

Werden Fördersysteme als Pufferspeicher benutzt (vgl. Abb. 4), werden die Stückgüter teilweise im Staubetrieb gefördert. Dabei erhöht sich in der Staulänge $L_{S}$ die Zugkraft nach Gleichung 2 noch um den Anteil der Reibung zwischen Zugmittel und Stückgut (Reibungswert $\mu_{\mathrm{ZM}-\mathrm{SG}}$ ). Somit ergibt sich die Zugkraft aus:

$$
\mathrm{F}_{\mathrm{Z}}=\mathrm{L}\left(\mathrm{q}_{\mathrm{SG}}+\mathrm{q}_{\mathrm{ZM}}\right) \cdot \mathrm{g} \cdot \mu_{\mathrm{ZM}-\mathrm{SFE}}+\mathrm{L}_{\mathrm{S}} \cdot \mathrm{q}_{\mathrm{SG}} \cdot \mathrm{g} \cdot \mu_{\mathrm{ZM}-\mathrm{SG}}
$$

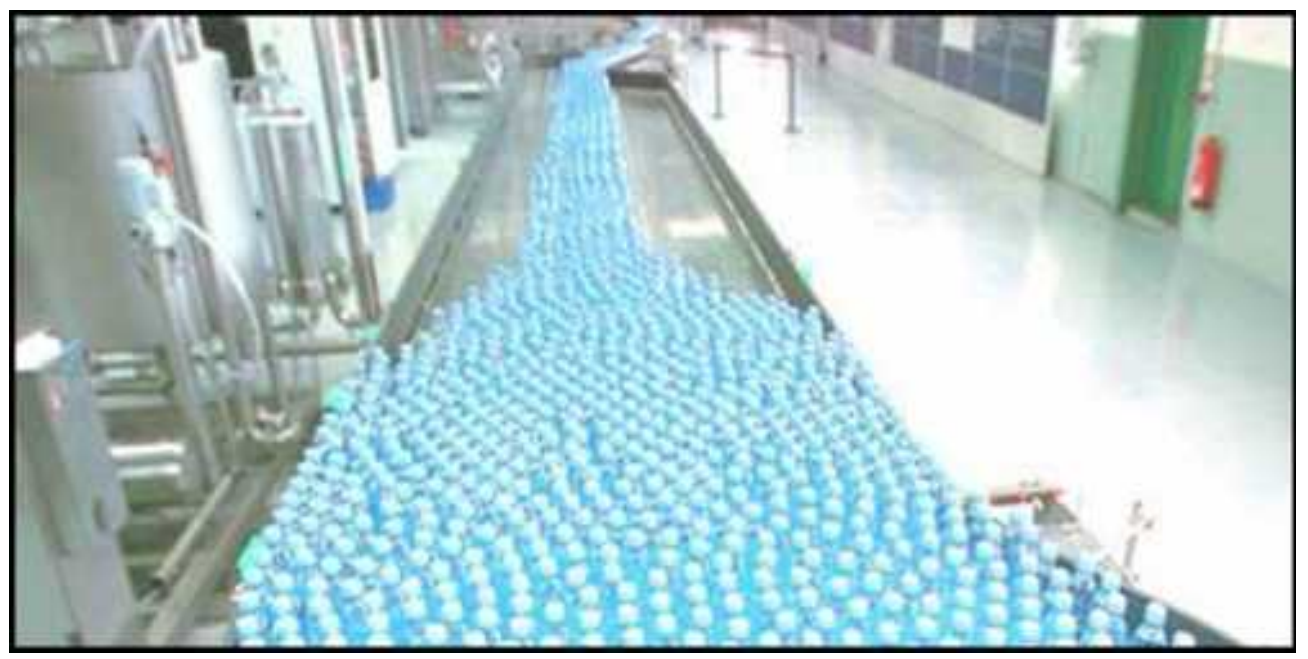

Abbildung 4: Pufferspeicher - Staubetrieb

Der Reibungswert zwischen Zugmittel und Stückgut hat im Staubetrieb direkten Einfluss auf die maximale Förderstreckenlänge und auf die benötigte Antriebsleistung. Ferner erhöht sich mit steigendem Reibungswert die Reibungsenergie, die als Wärme an das System und die Umgebung abgegeben wird. Dies kann zu einer unzulässigen Erwärmung des Zugmittels oder des Stückgutes führen und somit einen Systemausfall verursachen. Des Weiteren kann durch die unzulässige Erwärmung die maximale Länge der Staustrecke begrenzt oder das zu fördernde Stückgut beschädigt werden. Für Fördersysteme die mit Staubetrieb arbeiten, muss demzufolge der Reibungswert zwischen Zugmittel und Stückgut so niedrig wie möglich gehalten werden.

In anderen Anwendungsfällen muss der Reibungswert zwischen Zugmittel und Stückgut besonders hoch sein. Dies ist z.B. bei Fördersystemen mit Steigstrecken der Fall (vgl. Abb. 5). 


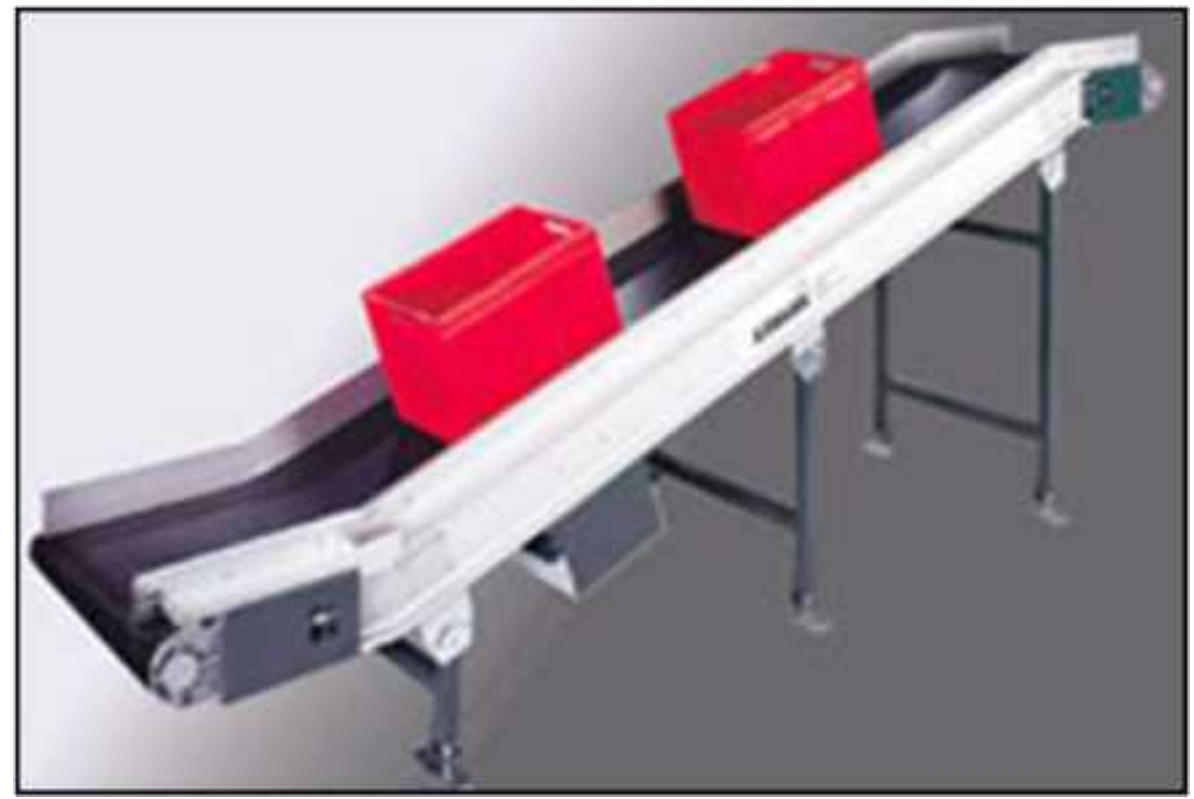

Abbildung 5: Steigstrecke eines Bandförderers

Für eine sichere Gutmitnahme muss die Mitnahmebedingung

$$
\tan \beta<\mu_{\mathrm{ZM}-\mathrm{SG}}
$$

erfüllt sein, wobei $\beta$ den Steigungswinkel der Steigstrecke und $\mu_{\mathrm{ZM}-\mathrm{SG}}$ den Haftreibungswert zwischen Zugmittel und Stückgut darstellt. Je größer der Reibungswert an dieser Stelle ist, desto größer kann der Steigungswinkel des Fördersystems werden. Dieser hohe Reibungswert ist notwendig, da die Realisierung eines geforderten Höhenunterschiedes bei geringem Steigungswinkel beträchtlichen Platz erfordert.

\subsection{Reibung zwischen Zugmittel und Antriebselement}

Zugmittel wie Gurte oder Bänder werden kraftschlüssig, über Reibung, angetrieben. Dazu wird das Zugmittel um die treibende Scheibe oder Trommel geschlungen (vgl. Abb. 6).

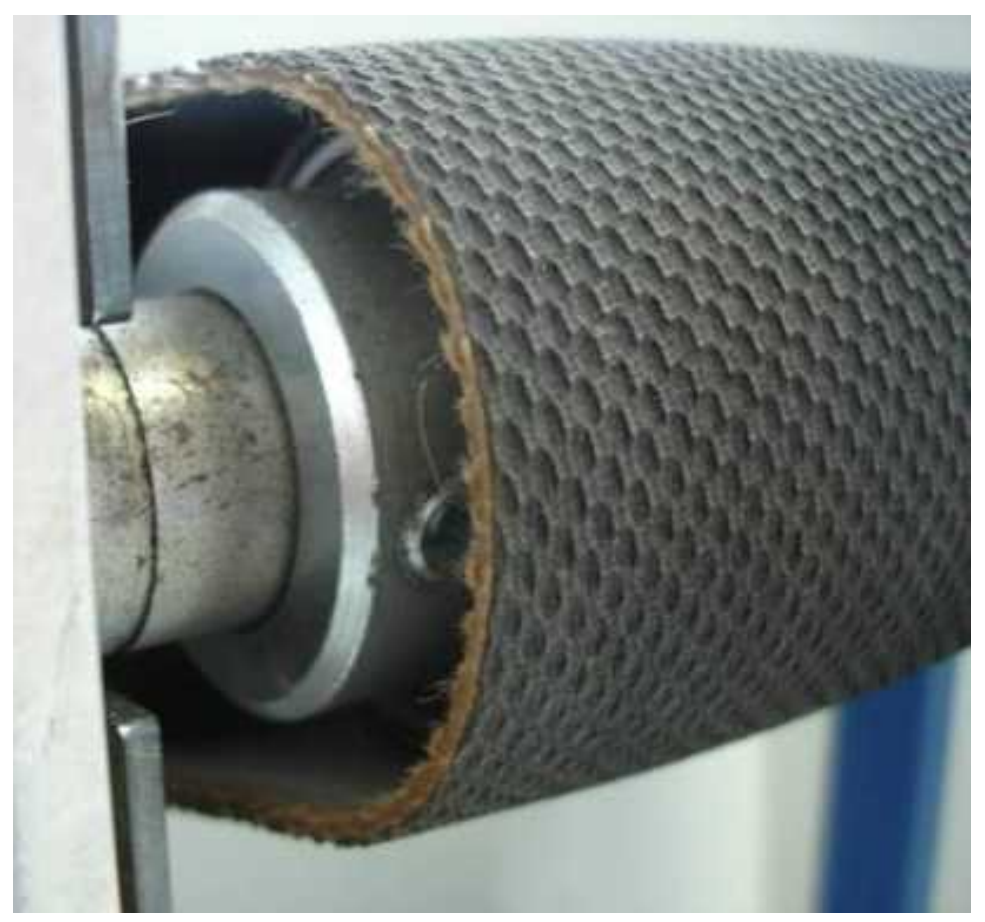

Abbildung 6: Gurtantrieb 
Die übertragbare Antriebsleistung eines Gurtförderers berechnet sich aus der Umfangskraft $\mathrm{F}_{\mathrm{U}}$ des Zugmittels und der Fördergeschwindigkeit $\mathrm{v}$ :

$$
\mathrm{P}_{\mathrm{A}}=\mathrm{F}_{\mathrm{U}} \cdot \mathrm{V}
$$

Die Umfangskraft ist die Differenz zwischen der Auflaufkraft $F_{1}$ und der Ablaufkraft $F_{2}$ des Zugmittels.

$$
\mathrm{F}_{\mathrm{U}}=\mathrm{F}_{1}-\mathrm{F}_{2}
$$

Aus der Eytelweinschen Bedingung:

$$
\mathrm{F}_{1}=\mathrm{F}_{2} \cdot \mathrm{e}^{\mu_{\mathrm{ZM}-\text { Antrieb }} \cdot \alpha}
$$

ergibt sich für die Auflaufkraft, d.h. für die maximal zu übertragende Zugkraft ein Maximum in Abhängigkeit des Reibungswertes $\mu_{\mathrm{ZM} \text {-Antrieb }}$ zwischen Zugmittel und Antriebsscheibe. Der Umschlingungswinkel $\alpha$ ist meist nicht variabel und beträgt in der Regel $\pi$. Um eine möglicht große Antriebsleistung auf den Gurt zu übertragen, sollte der Reibwert zwischen Antriebsscheibe und Zugmittel so groß wie möglich sein.

\subsection{Reibung - eine Systemeigenschaft}

Aus den dargestellten Beispielen ist erkennbar, dass für den Betrieb von Stückgutfördersystemen teils niedrige aber teils auch möglichst hohe Reibungswerte benötigt werden. Eine einfache Auswahl von geeigneten Materialien für die jeweiligen Bauteile des Förderers über Tabellen o.ä. ist nicht möglich. Reibungswerte sind keine Materialeigenschaften. Sie sind Systemeigenschaften und ergeben sich immer erst aus den Eigenschaften der am Reibungsvorgang beteiligten Systemkomponenten und aus dem Beanspruchungskollektiv. Zu den Eigenschaften der Systemkomponenten zählen neben dem Material z.B. die Härte und die Rauhigkeit der Oberflächen. Auch das Vorhandensein eines Mediums zwischen den beiden Kontaktkörpern, Schmierstoffe oder Schmutzpartikel, und die Eigenschaften des Umgebungsmediums, z.B. Feuchtigkeit der Luft, beeinflussen den Reibungswert. Zu beachten ist ferner, dass die am Reibungsvorgang beteiligten Elemente der Fördersysteme häufig aus Kunststoff bestehen und Kunststoffe ihre Eigenschaften in Abhängigkeit der Temperatur, der Luftfeuchtigkeit und der Zeit verändern. Zum Beanspruchungskollektiv werden alle äußeren wirkenden Einflüsse gezählt. Dazu gehören neben der Normalkraft auch die Art und Geschwindigkeit der Relativbewegung, die Temperatur und die Beanspruchungsdauer. (vgl. [Czichos06])

Auf Grund der großen Anzahl an bekannten aber teils auch unbekannten Faktoren und Parametern kann der Reibungswert nicht ausreichend genau vorhergesagt oder gar berechnet werden. Somit ist immer eine experimentelle Bestimmung dieser notwendig.

\section{Methoden zur Messung von Reibungswerten}

An einen Prüfstand zur Bestimmung von Reibungswerten werden hohe Anforderungen gestellt, die sich aus dem Systemgedanken der Tribologie ergeben. Er sollte sowohl die Systemstruktur als auch das in der Praxis herrschende Beanspruchungskollektiv möglichst realitätsnah abbilden können. Ferner sollte der Prüfstand sowohl Probeköper kleinerer und mittlerer Abmessung als auch komplette Bauteile oder Baugruppen prüfen können. Diesen Forderungen kommen bisherige Prüfstände nicht nach. Mit dem weit verbreiteten Modellprüfstand Stift-Scheibe können nur Probekörper kleinster Abmessung getestet werden, deren spezifische Eigenschaften jedoch nicht den Eigenschaften der kompletten Bauteile entsprechen. Diese Art von Versuchstand ist somit für die praxisnahe Bestimmung von Reibungswerten vollkommen ungeeignet.

Eine weitere Art von Prüfstand zur Untersuchung von Reibungswerten ist in Abbildung 7 dargestellt. Dabei wird das zu untersuchende Zugmittel mit der Lauffläche nach oben zwischen Antriebs- und Umlenkscheibe eingespannt und läuft um (vgl. [Sumpf98]). Die Elemente des Stütz- und Führungssystems werden auf das Zugmittel aufgelegt, mit Massestücken belasten und die Reibungskraft gemessen, wobei Normalkraft und Geschwindigkeit variiert werden können. Der Versuchsstand bildet das real existierende tribologische System mit hoher Genauigkeit ab und ist somit für die Messung des Reibungswertes zwischen Zugmittel und Stütz- und Führungssystem bzw. Stückgut geeignet. Bedingung ist jedoch, dass ein endloses Zugmittel vorhanden ist. 


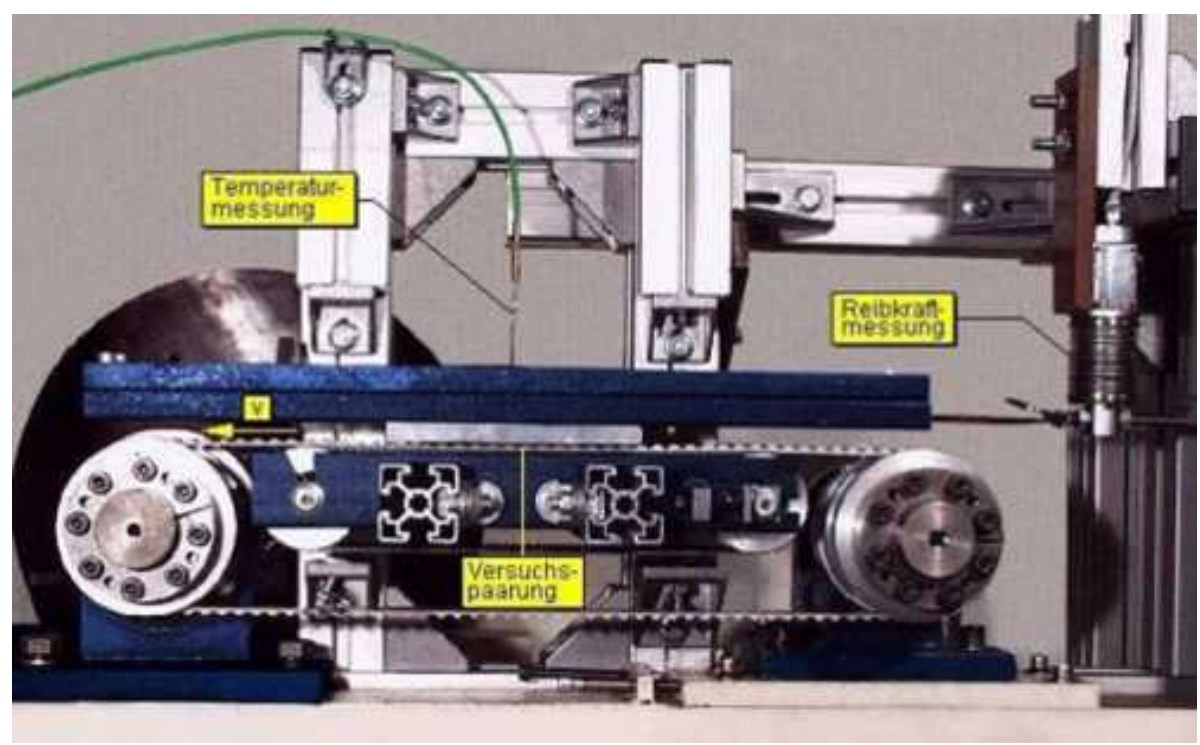

Abbildung 7: Versuchstand mit umlaufendem Zugmittel

Während der Entwicklung neuer Komponenten eines Fördersystems oder beim Test neuer Materialien ist jedoch oft kein endloses Zugmittel vorhanden, so dass diese Art von Versuchstand die notwendigen Entwicklungsschritte vom Probekörper über das Modell zum fertigen Bauteil nicht abdecken kann.

Aus diesem Grunde wurde an der TU Chemnitz ein neuer Reibungs- und Verschleißversuchsstand entwickelt (vgl. Abb. 8), mit dessen Hilfe Proben und Bauteile mit variablen Abmessungen und Formen untersucht werden können. Bei diesem Versuchsstand gleitet eine Oberprobe über die fest am Messtisch befestigte Unterprobe. Die Reibungskraft wird am Messtisch ermittelt. Um zwei Materialpaarungen gleichzeitig untersuchen zu können, sind am Versuchsstand zwei Messplätze realisiert worden.

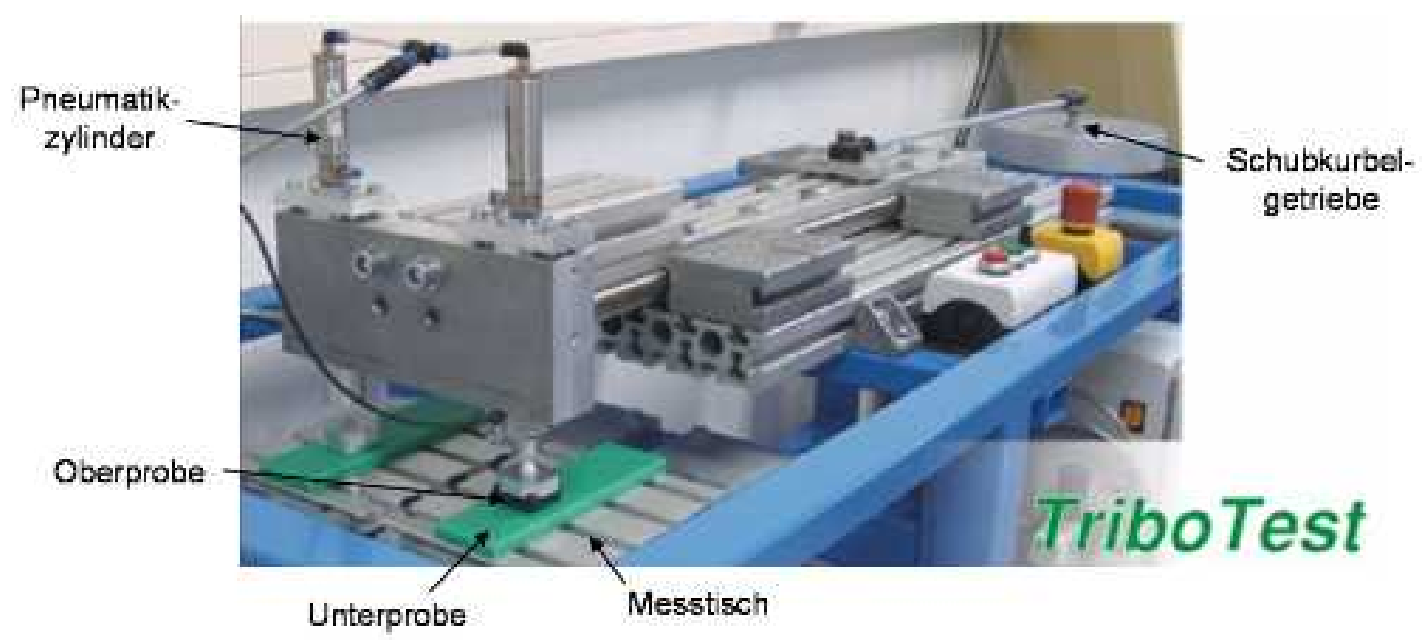

Abbildung 8: neuer Reibungs- und Verschleißprüfstand

Der Antrieb erfolgt oszillierend über ein Schubkurbelgetriebe. Somit wird eine kontinuierlich gleitende Bewegung erzeugt, deren Geschwindigkeit und Gleitweg stufenlos variiert werden können. Zu beachten ist, dass die Geschwindigkeit auf Grund der Kinematik des Getriebes nicht konstant ist, sie verläuft sinusförmig. Für die Beschreibung der Versuche wird deshalb die Durchschnittsgeschwindigkeit aus Gleitweg und Periodendauer angegeben.

Die Normalkraftaufbringung erfolgt pneumatisch. Durch die Regelung des Drucks am Zylinder kann die Normalkraft stufenlos verstellt werden. Die Kontrolle der Normalkraft während der Versuche erfolgt über Kraftmessdosen, welche jeweils in den Achsen der beiden Kolbenstangen integriert sind. Vorteile dieser Lösung sind geringe bewegte Massen sowie die Unabhängigkeit der Normalkraft vom Verschleißweg und von Unebenheiten der Probenoberflächen. 
Der Messtisch wird stirnseitig an Blattfedern gelagert (vgl. Abb. 9) und berührt das Gestell an keinen weiteren Punkten. Somit wird gewährleistet, dass in der Lagerung des Messtisches keine Reibung auftritt, die das Messergebnis beeinflussen kann.

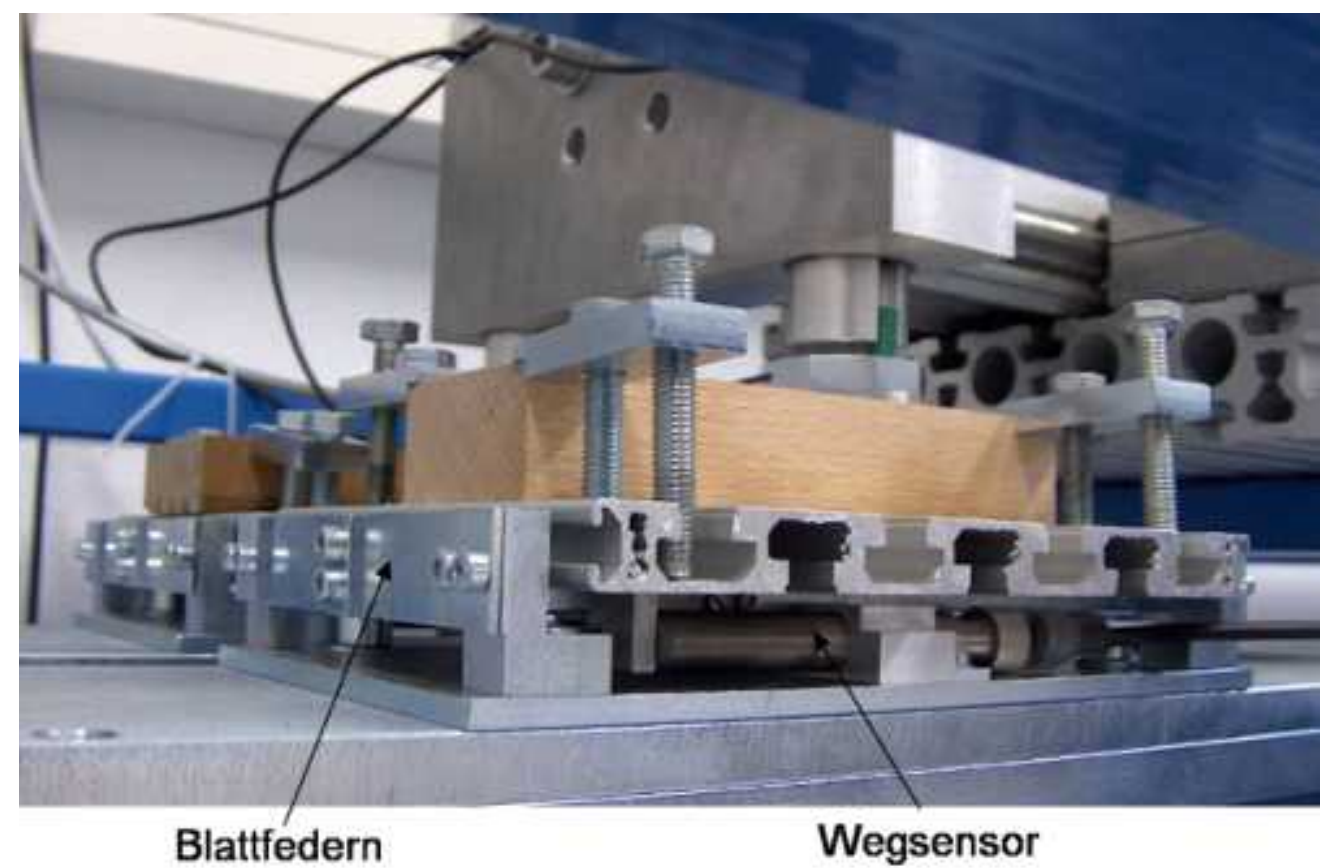

Abbildung 9: Lagerung Messtisch

Die beim Versuch auftretende Reibungskraft zwischen Ober- und Unterprobe wird auf den Messtisch und von dort auf dessen Lagerung, die Blattfedern übertragen. Das Durchbiegen dieser wird berührungslos über Induktionssensoren gemessen. Aus dem gemessenen Federweg und der bekannten Federkonstante der Blattfedern kann die Reibungskraft errechnet werden. Der Reibungswert ergibt sich als der Quotient aus der gemessenen Reibungskraft $F_{R}$ und der gemessenen Normalkraft $F_{N}$.

$$
\mu=\frac{F_{R}}{F_{N}}
$$

Der Verlauf des so ermittelten Reibungswertes ist in Abbildung 10 dargestellt.

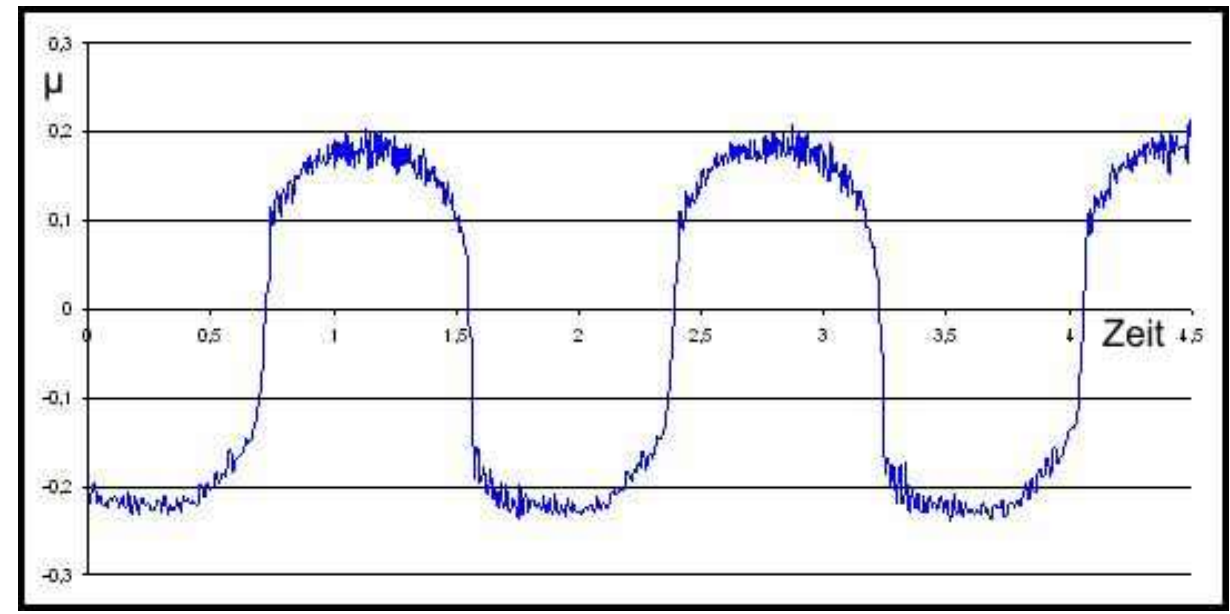

Abbildung 10: Reibungswert - Zeit - Diagramm 
Hierbei ist an den Umkehrstellen der oszillierenden Bewegung ein Sprung im Reibungswert zu erkennen. Der Grund dafür liegt in der Umkehr der Bewegungsrichtung, die eine Änderung der Richtung der Reibungskraft und somit eine Änderung des Vorzeichens des Reibungswertes bewirkt. Bei der Auswertung der Messergebnisse werden die Sprungstellen herausgefiltert und aus den verbleibenden Messwerten der Durchschnitt gebildet.

Als Probekörper für den Versuchstand können sowohl reale Bauteile wie Zahnriemen, Ketten, Gurte oder Gleitleisten als auch Probekörper in Form von Stiften oder Platten verwendet werden. Es ist kein umlaufendes Zugmittel notwendig, ein Produktmuster mit einer Länge von ca. 150mm ist ausreichend.

Der neue Versuchstand bietet die Möglichkeit sowohl Kurzzeitversuche über wenige Sekunden als auch Langzeitversuche über mehrere Wochen durchzuführen. Die Normalkraft sowie die Gleitgeschwindigkeit können stufenlos, auch während eines Dauerversuches, variiert werden. Die Auswertung der Versuche und die Erstellung eines Versuchsprotokolls, inklusive Verlaufsdiagramm des Reibungswertes, erfolgen über eine eigens entwickelte Software auf Basis von Visual Basic 6.

\section{Beispielhafte Messergebnisse des neuen Versuchsstandes}

In Abbildung 11 bis Abbildung 13 sind einige, mit Hilfe des neuen Versuchstandes ermittelte, Messergebnisse dargestellt. Dabei wurde als Unterprobe eine geschliffene Edelstahlplatte mit den Abmessungen 180 x 60 mm und als Oberprobe verschiedenstes Gleitschienenmaterial mit den Abmessungen 30 x 30 mm verwendet. Die geometrische Kontaktfläche betrug somit $900 \mathrm{~mm}^{2}$. Während der Versuche wurden die Normalkraft und die Gleitgeschwindigkeit variiert, sowie der Einfluss der Gleitdauer näher betrachtet. Alle anderen Einflussparameter wurden konstant gehalten.

In Abbildung 11 ist der zeitliche Verlauf des Reibungswertes verschiedener Gleitschienenmaterialien gegen geschliffenen Edelstahl dargestellt.

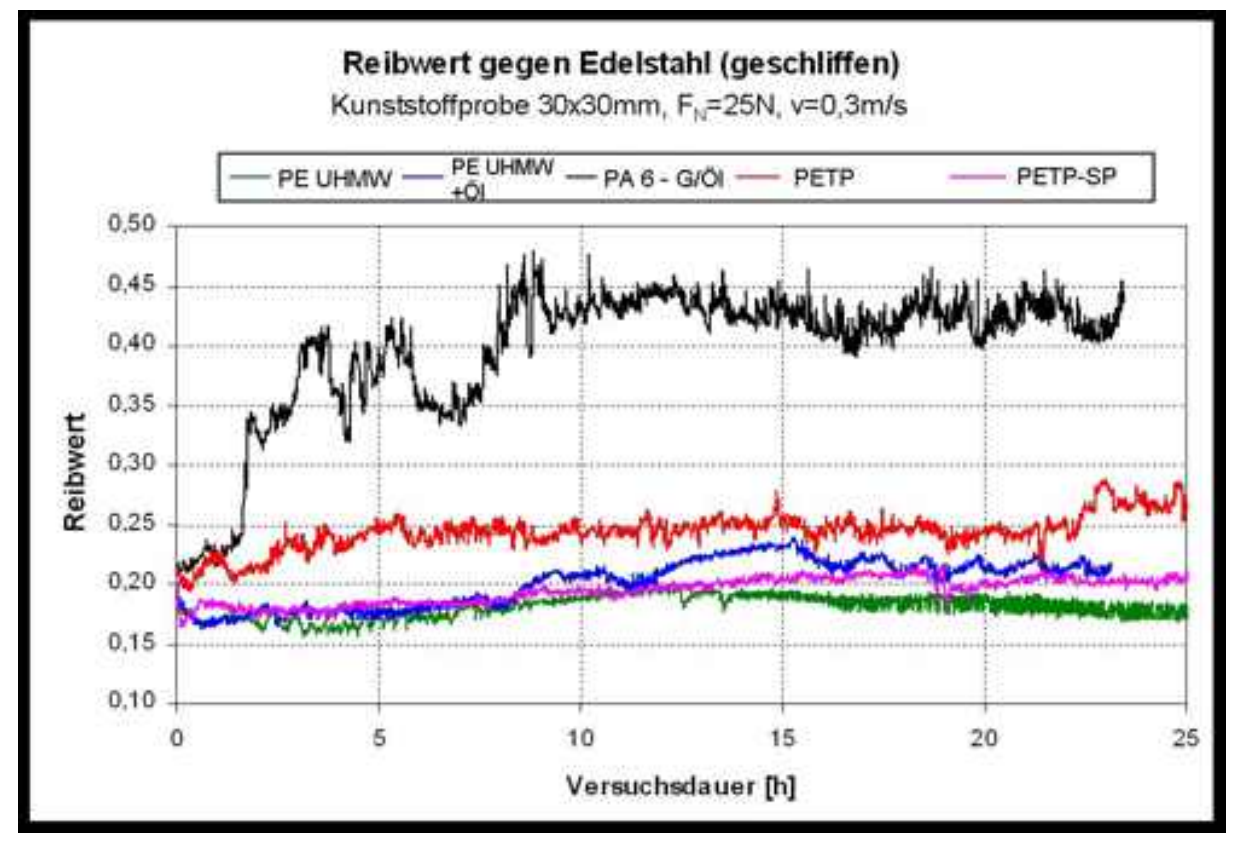

Abbildung 11: Verlauf des Reibungswertes über die Gleitdauer

Deutlich erkennbar ist dabei das auffällige Verhalten des Materials PA 6 - G/Öl. Im Vergleich zu allen anderen Materialpaarungen steigt der Reibungswert bereits nach kurzer Gleitdauer deutlich an und erreicht nach ca. 8,5 Stunden ein Maximum, welches dann bis zum Ende des Versuches gehalten wird. Der Reibungswert hat sich dabei gegenüber dem Startwert verdoppelt. Daraus lässt sich ableiten, dass Reibungswertmessungen immer über einen längeren Zeitraum durchgeführt werden müssen. Als vorteilhaft hat sich dabei eine Versuchsdauer von 24 Stunden erwiesen. Kurzzeitversuche können zu falschen Aussagen führen!

In Abbildung 12 sind die Langzeitreibungswerte (nach 24 Stunden) einer Kunststoffprobe aus PE UHMW gegen geschliffenen Edelstahl bei verschiedenen Gleitgeschwindigkeiten dargestellt. 
Der Geschwindigkeitseinfluss zeigt dabei im Bereich von 0 bis $0,5 \mathrm{~m} / \mathrm{s}$ einen degressiven Anstieg des Reibungswertes, d.h. mit steigender Geschwindigkeit wird die Zunahme des Reibungswertes geringer. Da die Einsatzbedingen von Fördersystemen schwanken können, sollten zur Dimensionierung dieser immer die Reibungswerte der höchsten zulässigen Geschwindigkeit verwendet werden.

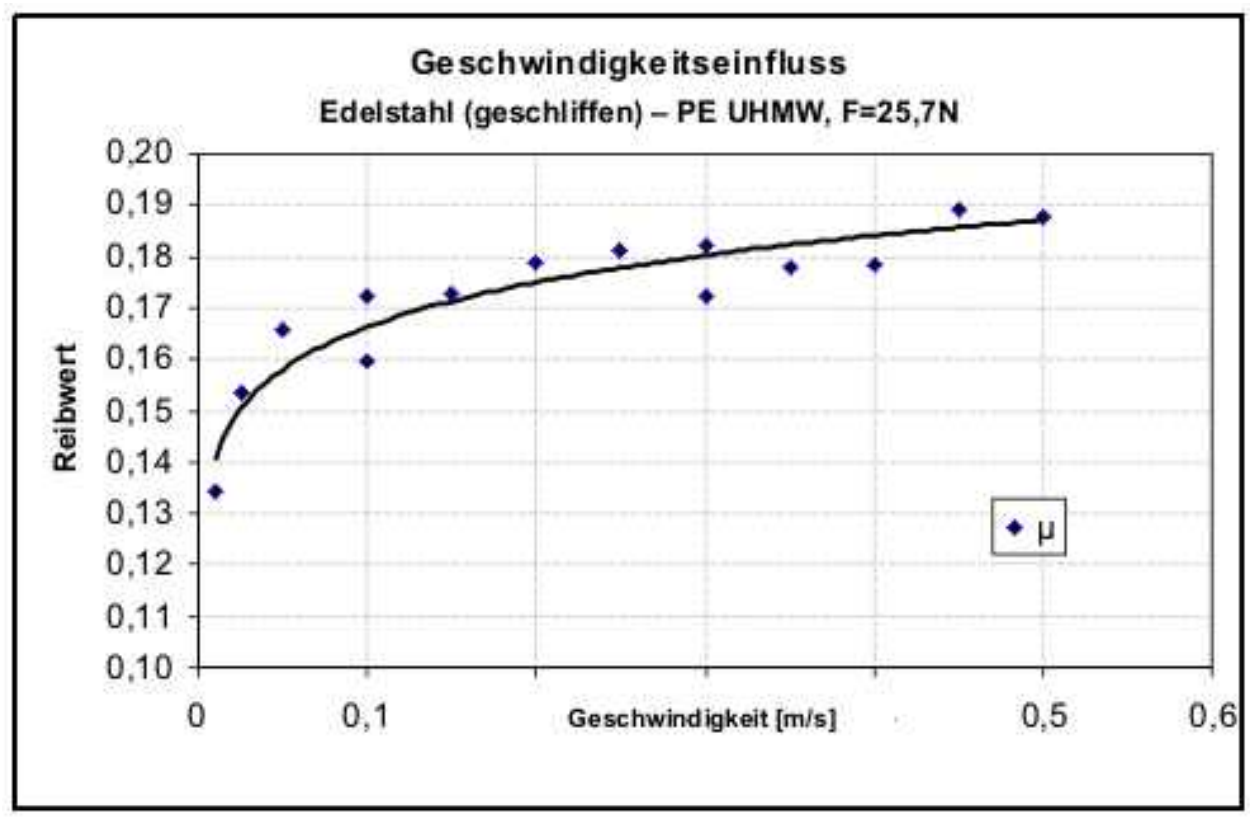

Abbildung 12: Einfluss der Geschwindigkeit auf den Reibungswert

In Abbildung 13 sind die Reibungswerte nach 24 Stunden einer Kunststoffprobe aus PE UHMW gegen geschliffenen Edelstahl in Abhängigkeit der Gleitgeschwindigkeit und der Normalkraft abgebildet. Auch hier ist erkennbar, dass der Reibungswert mit steigender Geschwindigkeit ansteigt. Der Verlauf des Reibungswertes in Abhängigkeit von der Normalkraft ist hingegen nicht einheitlich. Bei niedrigen Geschwindigkeiten fällt der Reibungswert mit steigender Normalkraft, bei mittleren Geschwindigkeiten bleibt er konstant und bei hohen Geschwindigkeiten steigt er an.

Die ansteigenden Reibungswerte bei hohen Geschwindigkeiten in Kombination mit hohen Normalkräften sind auf die Temperaturentwicklung in der Kontaktzone zwischen Ober- und Unterprobe zurückzuführen. Die durch den Reibungsvorgang entstehenden hohen Temperaturen verändern die Oberfläche des thermisch sensibleren Kontaktpartners, hier PE UHMW. Sie schmilzt teilweise auf und führt somit zu höheren Reibungswerten. 


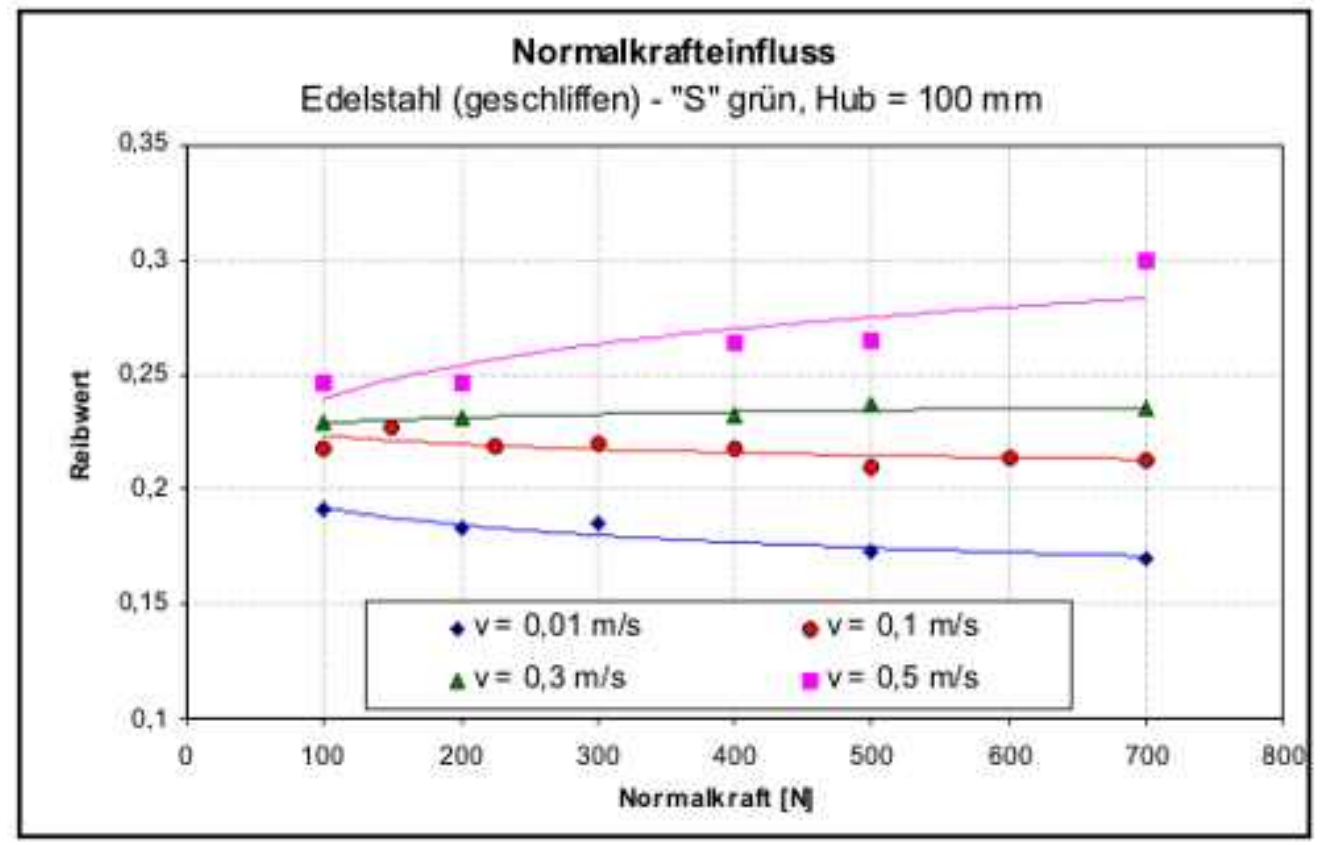

Abbildung 13: Einfluss der Normalkraft auf den Reibungswert

\section{Zusammenfassung und Ausblick}

Mit dem neu an der TU Chemnitz entwickelten Reibungsprüfstand können erstmals Reibwerte von Probekörpern und kompletten Bauteilen oder Baugruppen kleinerer bis mittlerer Größe auf einem Versuchsstand bestimmt werden. Dabei können die beiden wichtigsten technischen Beanspruchungsparameter, die Geschwindigkeit und die Normalkraft, stufenlos verstellt werden. Erste Untersuchungen auf dem neuen Versuchsstand haben gezeigt, dass vor allem bei Kunststoffen die Reibungswerte über die Zeit stark schwanken können. Aus diesem Grund sollten praxisrelevante Reibungswertmessungen nur mit Dauerversuchen durchgeführt werden.

Während der weiteren Entwicklung des Versuchsstandes soll in diesen eine Klimakammer integriert werden. Mit deren Hilfe können erstmals bei Bauteilen und Baugruppen die Abhängigkeiten des Reibungswertes von der Umgebungstemperatur und der Luftfeuchte gemessen werden.

\section{Literatur}

[Auerbach06]

Auerbach, P.: Zur Beanspruchung und Lebensdauer raumgängiger Gleitketten aus

[Czichos06] Kunststoffen. Dissertation, TU Chemnitz, 2006.

[Sumpf98] Czichos, Horst ; Habig, Karl-Heinz: Tribologie-Handbuch Reibung und Verschleiß.Vieweg, Wiesbaden, 2006 Sumpf, J.; Nendel, K.: Führungsschienen für Stetigförderer - Kunststoffe im Beanspruchungstest. Hebezeuge und Fördermittel, Berlin 38 (1998) 5, S. 239-242 